\title{
Study of risk factors and prevalence of invasive candidiasis in a tertiary care hospital
}

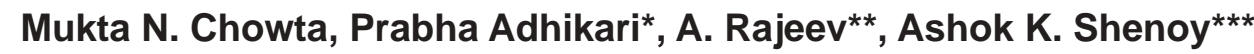

Introduction: The frequency of invasive mycoses has increased dramatically during the past two decades owing to medical advances such as intensive cancer chemotherapy, broad-spectrum antimicrobial therapy, invasive medical devices, organ transplantation, human immunodeficiency virus (HIV) disease epidemic and an expanding aging population. There were few Indian studies regarding the incidence and risk factors for candidemia. Hence the aim of this work was to evaluate the changes in the prevalence of candidemia and invasive candidiasis in a tertiary care hospital and also to assess the risk factors and predictors of mortality Materials and Methods: Nonsystematic review of patients with candidemia/invasive candidiasis was done during the period 1999 to 2004. All in-patients who had shown signs and symptoms of nosocomial blood stream infection were screened for candidial infection. Among these, 29 patients had candidemia/invasive candidiasis. Demographic and clinical data of these patients were recorded on a standardized form, which included age, sex, site of isolation, infectious diagnosis, underlying conditions, predisposing factors, catheter status and clinical outcome. The data were collected during the years of 1999 to 2004, which is divided into two time periods (1999-2001 and 2002-2004). Data collected during these different time spans are compared with each other. Results: A total of 255 patients were screened during the study period. Among these, 100 patients were screened during the period 1999-2001 and 155 patients were screened during the year 20022004. Out of these patients, 29 showed positive cultures in blood or other sterile site (ascitic fluid, bronchial aspirate and urine from suprapubic puncture). Out of these, 24 were males and five were females. The most common risk factor was use of intravenous canulae (62.1\%), followed by prolonged use of antibiotics (34.5\%) and HIV infection (24.1\%). There were no statistically significant differences in the risk factors during the two different study periods. Candida was mainly isolated from blood (75.9\%). Other sources included ascitic fluid (10.4\%), bronchial aspirate (3.4\%), sputum (3.4\%) and urine (6.9\%). Distributions of sources were comparable during the two study periods. Candida albicans, Candida tropicalis and Candida parapsilosis caused $89.7 \%, 3.4 \%, 6.9 \%$ of the candidemia episodes respectively. The overall mortality was $51.7 \%$. Conclusion: The present study emphasizes the importance of candidemia among hospitalized patients. Continued surveillance of candidemia will be important to track trends of this serious infection and to document changes in its epidemiological features. More active screening in high-risk groups should be done to avoid diagnostic delay. Risk factors like prolonged use of multiple antibiotics, central venous catheters, mechanical ventilation and prolonged hospital stay should be restricted whenever possible. Timely use of antiretroviral drugs and other measures to improve the immunity of HIV patients may help to decrease the incidence of candidemia in this patient population.

Key words: C. albicans, candidiasis, human immunodeficiency virus infection, risk factors

\section{From:}

Departments of Pharmacology, ${ }^{*}$ Medicine, ${ }^{* *}$ Community Medicine,

${ }^{* * *}$ Pharmacology, Kasturba Medical College, Mangalore, India
Correspondence

Dr. Mukta N Chowta, Department of Pharmacology, Kasturba Medical College, Mangalore - 575001, India. E-mail: muktachowta@yahoo.co.in 


\section{Introduction}

The frequency of invasive mycoses has increased dramatically during the past two decades owing to medical advances such as intensive cancer chemotherapy, broad-spectrum antimicrobial therapy, invasive medical devices, organ transplantation, human immunodeficiency virus (HIV) disease epidemic and an expanding aging population. Mortality due to fungal infection increased three to four fold between 1980 and 1997, with mycoses ranking as the seventh leading cause of death in 1997 compared with tenth in 1980. Much of the increase during this period was due to HIV associated cryptococcosis and pneumocystosis; the rates of occurrence of both have since declined dramatically with the advent of more effective antiretroviral therapy and antifungal prophylaxis. Among patients who are not infected with HIV, however, invasive candidiasis and aspergillosis remain significant causes of morbidity and mortality. Current data from the surveillance and control of pathogens of epidemiological importance (SCOPE) surveillance system confirms that Candida species have become the fourth leading cause of blood stream infections. ${ }^{[1]}$ Candidemia is associated with increased cost and attributable mortality of $38 \% .^{[2]}$ Although Candida albicans is the most frequent organism encountered, a number of reports have documented infections caused by C. tropicalis, C. glabrata, C. parapsilosis and C. krusei. ${ }^{[3,4]}$ Non-albicans Candida species and other filamentous fungi also have emerged as significant pathogens in recent years. ${ }^{[5]}$ Careful epidemiologic studies have identified intravascular catheters, broad-spectrum antibiotics therapy, mucosal colonization, neutropenia, previous surgical procedures (particularly complicated abdominal surgery), total parenteral nutrition and concomitant bacteremia as significant risk factors for invasive candidal infection. ${ }^{[6-8]}$

There were few Indian studies regarding the incidence and risk factors for candidemia. Hence the aim of this work was to evaluate the changes in the prevalence of candidemia and invasive candidiasis in a tertiary care hospital and also to assess the risk factors and predictors of mortality.

\section{Metarials and Methods}

Nonsystematic review of patients with candidemia/ invasive candidiasis was done during the period 1999 to 2004. All in-patients who had shown signs and symptoms of nosocomial blood stream infection were screened for candidial infection. Among these, 29 patients had candidemia/invasive candidiasis. Demographic and clinical data of these patients were recorded on a standardized form which included age, sex, site of isolation, infectious diagnosis, underlying conditions, predisposing factors, catheter status, steroid treatment, chemotherapy, culturing and clinical outcome. The criterion for inclusion in the study was age above 12 years with positive fungal culture in hospitalized patients. Children below the age of 12 years and patients with less than $48 \mathrm{~h}$ of hospital stay were excluded. The major reason for their hospital admission was some other underlying condition other than candidemia. The data were collected during the years of 1999 to 2004. This period is divided into two different time spans i.e., 19992001, 2002-2004. Data collected during these different time spans are compared with each other. Strains of candida isolated from blood or other normally sterile sites in hospital laboratories were sent to central laboratory for further analysis.

Blood culture was performed using the automated blood culture system (Bact/alert). Five $\mathrm{ml}$ of blood/sterile body fluid was inoculated into Myco F Lytic medium. Culture bottles were loaded into the instrument and remained there for seven days or until designated positive. Subsequent subculture was done for speciation. Patients who showed positive cultures analyzed for predisposing factors for candidemia. These factors were analyzed for their association with mortality.

Statistical analysis was performed using Chi square test, fisher's exact test and student 't' tests

\section{Results}

A total of 255 patients were screened during the study period. Among these, 100 patients were screened during the period 1999-2001 and 155 patients were screened during the year 2002-2004. Out of these patients, 29 showed positive cultures in blood or other sterile site (ascitic fluid, bronchial aspirate and urine from suprapubic puncture). Out of these, 24 were males and five were females.

Table 1 summarizes the distribution of underlying diseases. Table 2 shows the risk factors within this population of patients with candidemia. At the onset of 
Table 1: Distribution of underlying diseases

Underlying diseases

Human immunodeficiency virus

Neutropenia

Chronic obstructive airway disease

Pancreatitis

Infective endocarditis

Tuberculosis

Typhoid fever

Malignancy

Burns

Sepsis

Poisoning

Diabetes mellitus

Fracture

\begin{tabular}{cc} 
& Year \\
\hline $\mathbf{1 9 9 9 - 2 0 0 2 ( n = 1 4 )}$ & $\mathbf{2 0 0 3 - 2 0 0 4 ( n = 1 5 ) ( \% )}$ \\
$4(28.6)$ & $3(20)$ \\
$1(7.1)$ & 0 \\
0 & $1(6.7)$ \\
$1(7.1)$ & 0 \\
$1(21.4)$ & 0 \\
$2(14.3)$ & $1(6.7)$ \\
$1(7.1)$ & $1(6.7)$ \\
$1(14.3)$ & $1(6.7)$ \\
$1(7.1)$ & 0 \\
0 & $4(26.7)$ \\
$1(14.3)$ & $1(6.7)$ \\
0 & $2(13.3)$ \\
$1(7.1)$ & $1(6.7)$ \\
\hline
\end{tabular}

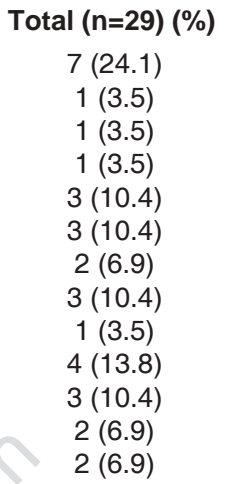

\section{Table 2: Distribution of risk factors for candidiasis}

\begin{tabular}{|c|c|c|c|}
\hline \multirow[b]{2}{*}{ Risk factors } & \multicolumn{2}{|c|}{ Year } & \multirow[b]{2}{*}{ Total $(n=29)(\%)$} \\
\hline & $1999-2002(n=14)$ & $2003-2004(n=15)(\%)$ & \\
\hline Prolonged antibiotics & $8(57.1)$ & $8(53.3)$ & $10(34,5)$ \\
\hline IV canula & $8(57.1)$ & $10(66.7)$ & $18(62.1)$ \\
\hline Prolonged fever & $2(14.3)$ & 0 & $2(6.9)$ \\
\hline Steroid use & $3(21.4)$ & $1(6.7)$ & $4(13.8)$ \\
\hline Pneumonia & $2(14.3)$ & $2(13.3)$ & $4(13.8)$ \\
\hline Tuberculous meningitis & $1(7.1)$ & $1(6.7)$ & $2(6.9)$ \\
\hline Surgery & $1(7.1)$ & $3(20)$ & $4(13.8)$ \\
\hline Ventillator & $3(21.4)$ & $1(6.7)$ & $4(13.8)$ \\
\hline Fracture & $1(7.1)$ & $1(6.7)$ & $2(6.9)$ \\
\hline Organ failure & $2(14.3)$ & $2(13.3)$ & $4(13.8)$ \\
\hline
\end{tabular}

candidemia, all patients had at least one predisposing factor. The most common risk factor was use of intravenous canulae $(62.1 \%)$, followed by prolonged use of antibiotics (34.5\%). But the careful scrutiny of cases showed that IV canulae might be the risk factor in only two patients who had typhoid fever. Other patients with IV canulae had various other risk factors. Most common underlying disease was HIV infection (24.1\%). There were no statistically significant differences in the risk factors during the two different time periods. Most of the patients had multiple risk factors. Out of the 27 patients, nine had two different risk factors, six had three risk factors, seven had four risk factors, five had five risk factors, one had six and another one had seven risk factors.

Candida was mainly isolated from blood (75.9\%). Other sources included ascitic fluid (10.4\%), bronchial aspirate (3.4\%), sputum (3.4\%) and urine (6.9\%). Distributions of sources were comparable during the two time periods [Table 3].

Table 4 shows the distribution of candida species among the culture positive patients. Candida albicans, Candida tropicalis and Candida parapsilosis caused $89.7 \%$, $3.4 \%, 6.9 \%$ of the candidemia episodes respectively. Distributions of species were similar during the two time periods except that Candida tropicalis was isolated in one patient during the period 2002-2004.

The overall mortality was $51.7 \%$. There was no statistically significant difference in the outcome during the two time periods [Table 5]. Risk factors in patients

Table 3: Source for fungal isolation

\begin{tabular}{|c|c|c|c|}
\hline \multirow[b]{2}{*}{ Source } & \multicolumn{2}{|c|}{ Year } & \multirow[b]{2}{*}{ Total $(n=29)(\%)$} \\
\hline & $1999-2002(n=14)(\%)$ & $2003-2004(n=15)(\%)$ & \\
\hline Ascitic fluid & 0 & $3(20)$ & $3(10.4)$ \\
\hline Blood & $13(92.9)$ & $9(60)$ & $22(75.9)$ \\
\hline Bronchial aspirate & 0 & $1(6.7)$ & $1(3.4)$ \\
\hline Sputum & $1(7.1)$ & 0 & $1(3.4)$ \\
\hline Urine & 0 & $2(13.3)$ & $2(6.9)$ \\
\hline
\end{tabular}




\section{Table 4: Species of Candida}

\begin{tabular}{|c|c|c|c|}
\hline \multirow[b]{2}{*}{ Species } & \multicolumn{2}{|c|}{ Year } & \multirow[b]{2}{*}{ Total $(n=29)(\%)$} \\
\hline & $1999-2002(n=14)(\%)$ & $1999-2002(n=15)(\%)$ & \\
\hline C. albicans & $13(92.9)$ & $13(86.7)$ & $26(89.7)$ \\
\hline C. parapsilosis & $1(7.1)$ & $1(6.7)$ & $2(6.9)$ \\
\hline C. tropicalis & 0 & $1(6.7)$ & $1(3.4)$ \\
\hline
\end{tabular}

\section{Table 5: Outcome of candidiasis}

\begin{tabular}{|c|c|c|c|}
\hline \multirow[b]{2}{*}{ Outcome } & \multicolumn{2}{|c|}{ Year } & \multirow[b]{2}{*}{ Total $(n=29)(\%)$} \\
\hline & 1999-2002 (\%) & 2003-2004 (\%) & \\
\hline $\begin{array}{l}\text { Died } \\
\text { Responded }\end{array}$ & $\begin{array}{l}7(50) \\
7(50)\end{array}$ & $\begin{array}{l}8 \text { (53.3) } \\
7(46.7)\end{array}$ & $\begin{array}{l}15(51.7) \\
14(48.3)\end{array}$ \\
\hline
\end{tabular}

Fisher's exact test $(P=0.576)$

who died during the two time periods were comparable. Mortality was seen mainly in patients with HIV as the underlying disease and in those who had multiple risk factors. There was no statistically significant difference in age distribution of patients during the two time periods. Comparison of age versus outcome (mortality) also did not show any statistical significance.

\section{Discussion}

The present study emphasizes the importance of candidemia among hospitalized patients. Candidemia is not only associated with a significant mortality but also extends the duration of hospital stay and increases the cost of medical care. Patients with candidemia usually present with acute septic syndrome that is indistinguishable from bacteremia, but they may also exhibit a more indolent course manifested by fever of unknown origin. Major risk factors for candidemia include intravascular catheters, broad-spectrum antibiotics and immunosuppression.

The present study highlights the importance of candidemia among patients infected with HIV, in addition to the high risk groups such as, patients with IV canula, parenteral antibiotics, malignancy, surgery etc. The predisposing factors and underlying diseases observed in this study are comparable to those observed by others. ${ }^{[6-9]}$ Though the study data suggest IV canulae as the major risk factor, careful scrutiny of cases showed that IV canulae may be the important risk factor in only two patients with typhoid fever. In all other cases patients had other risk factors also. Abdominal surgery was one of the important risk factor, which indicates that source of candida is endogenous and probably the gut which have been colonized.
Our study confirms the importance of surgery, cancer, HIV infection and hematologic malignancies as factors contributing to nosocomial candidemia. Our patient population included 7 HIV patients $(24.1 \%)$. In the United States the proportion of candidemia cases with HIV infection to all candidemia cases varied from $10 \%$ to $15 \%$. ${ }^{[10,11]}$ The contribution of HIV infection as a predisposing risk factor for candidemia is further emphasized by a report from Italy, where Candida spp. was the third most common cause of nosocomial blood stream infections in HIV patients. ${ }^{[12]}$ In France, among cases of nosocomial candidemia, $13 \%$ of patients were reported to have HIV infection during 1990-1995 in one institution. ${ }^{[13]}$

Central venous catheter as a risk factor for candidemia, has been discussed in many studies. ${ }^{[8,14]} A$ strong consensus has been emerged recently on the fact that intravenous lines should be withdrawn from candidemic patients whenever possible. ${ }^{[15]}$ More recently, a prospective multicenter study from a tertiary care hospital in Brazil demonstrated that non-removal of the central venous catheter, in addition to the older age, were the only factors associated with an increased risk of death. ${ }^{[16]}$

Use of multiple antibiotics will suppress the bowel flora. ${ }^{[17,18]}$ Restoration of normal gut flora is the fundamental prerequisite for the control of fungal infections. This requires the restricted use of broad-spectrum antibiotics, which are known to affect the gut ecology.

Our result shows that $C$. albicans remains the Candida species most frequently implicated in nosocomial candidiasis with an overall frequency of $89.7 \%$. We did 
not identify any shift towards non- $C$. albicans species, in contrast to several reports from the United States, Australia and Europe..$^{[10,19-21]}$ The male dominance which was observed in the present study is similar to that found in previous reports. ${ }^{[10,22-24]}$ Differences in candidemia rates between countries may also be attributable to differences in the study population, the prevalence of HIV infection in the study population and variations in patterns of healthcare delivery and clinical practices, including the frequency of using blood cultures in diagnosis. The differences may also be explained by differences in antibiotic use patterns and resistance situation.

The overall mortality was $51.7 \%$. Despite antifungal treatment, the mortality of candidemia was high which indeed may reflect the severity of the already existing underlying diseases at the onset of candidemia, as well as direct mortality due to candidemia itself. The attributable mortality could not be assessed in the present study, as the study design did not include the matched control group. Study by Leleu et al. showed an attributable mortality as $31 \%$ in candidemia. ${ }^{[25]}$ This study was done by matching the patients with systemic candidemia to those without the evidence of systemic candidemia, but with similar underlying disease. Similarly, in an another study, Gudlauqsson et al., showed attributable mortality as $38 \%$ in nosocomial candidemia. ${ }^{[26]}$ Hence systemic candidemia is associated with high crude and attributable mortality which is much higher than that expected from underlying disease alone. Optimizing management of this infection is therefore imperative. Risk of death increases, if the treatment is delayed. Hence the empirical antifungal therapy, early in the course of suspected systemic candidiasis may improve the survival.

To a greater extent than for other fungi, treatment of candidemia can now be guided by in-vitro susceptibity testing. However, susceptibility testing of fungi is not considered a routine testing procedure in many laboratories and is not promptly available or is not universally considered as the standard of care. Knowledge of the infecting species, however, is highly predictive of likely susceptibilities and can be used as a guide to therapy. Susceptibility testing is especially helpful in deep infections due to nonalbicans species where the possibility of microbiological resistance must be considered. ${ }^{[27]}$
Amphotericin B based preparations, azole antifungals and the echinocandin antifungal agents play a role in the treatment. Choice of therapy is guided by weighing the greater activity of amphotericin $\mathrm{B}$ based preparations and echinocandin antifungal agents for some non albicans species against the ready availability of oral and parenteral formulations for azole antifungal agents. ${ }^{[27-28]}$ Flucytosine has activity against many isolates of candida but is infrequently used. Use of amphotericin may be complicated by dose limiting nephrotoxicity and reducing the dose may compromises with clinical efficacy. Liposomal preparations of amphotericin are less nephrotoxic, but highly expensive. Flucanozole has excellent safety profile, but Candida species other than C. albicans, $C$. glabrata may become resistant to flucanozole during the course of treatment. In addition, there has been a major shift in the past decade towards nonalbicans species, which now account for half of cases of candidemia. Identification of candida species is thus important in this regard. The recent development of echinocandin has resulted in a new therapeutic alternative to amphotericin $B$ and flucanozole for the primary treatment of candidemia and other forms of deeply invasive candidemia. ${ }^{[29]}$

Prophylactic strategies are useful if the risk of a target disease is sharply elevated in a readily identified patient group. The role of fluconazole prophylaxis is wellestablished in neutropenic patients; however, among patients without neutropenia, such as surgical ICU patients, this role is less definitive. ${ }^{[30]}$ The emergence of non- $C$. albicans species as agents of candidemia has been attributed to the increasing use of azole drugs and resulting selection of species less susceptible to these agents. Role of azole chemoprophylaxis in development of drug resistance in $C$. albicans and in emergence of less susceptible fungal pathogens needs to be examined. Clinical practices in prophylaxis policies may vary a great deal between institutions and countries. While the prophylaxis effectively reduces the incidence of infections caused by fluconazole-sensitive species, the drug has an impact on the distribution of causative Candida species. ${ }^{[30]}$

Present study included retrospective analysis of medical records from an individual hospital, which may not be representative of all hospitals serving a community. Analysis of population based laboratory surveillance may provide better understanding of epidemiological features 
of candidemia, its incidence and species distribution. This may also help to avoid bias that results from including only selected referral institutions.

The prevention of nosocomial infections is an important aspect of patient care, particularly in high-risk areas such as intensive care units. Local hospital leadership needs to develop easily defined infection control policies that are evidence-based. These infection-control policies also require the presence of a dedicated group of infectioncontrol practitioners to provide education, collect surveillance data and oversee the implementation of the local infection-control plan.

Treatment of underlying diseases, removal of possible risk factors and appropriate treatment with antifungals are all important steps in the management of systemic fungal infections. Although a number of risk factors for candidemia have been identified, consensus on preventive measures has not been achieved to date. Suggested methods for managements of central or peripheral IV catheters are still controversial. Strategies such as decreasing the number of antibiotics prescribed and azole chemoprophylaxis for high-risk group have been suggested, but not been widely implemented. Though the panel of experts reviewed the management and prevention of candidemia and developed consensus in this regard, data available to formulate these recommendations were limited and controversial. The effectiveness of prevention measures for candidemia, as well as cost- effectiveness and potential complications of such measures, require further evaluation. Continued surveillance of candidemia will be important to track trends of this serious infection and to document changes in its epidemiological features. More active screening in high risk groups should be done to avoid diagnostic delay. Risk factors like prolonged use of multiple antibiotics, central venous catheters, mechanical ventilation and prolonged hospital stay should be restricted whenever possible. Timely use of antiretroviral drugs and other measures to improve the immunity of HIV patients may help to decrease the incidence of candidemia in this patient population. Continued surveillance of candidemia will be important to document changes in epidemiological features of candidemia and antifungal susceptibilities.

\section{References}

1. Rangel-Frausto MS, Wiblin T, Blumberg HM, Saiman L, Patterson J,
Rinaldi M, et al. National epidemiology of mycoses survey (NEMIS): Variations in rates of bloodstream infections due to Candida species in seven surgical intensive care units and six neonatal intensive care units. Clin Infect Dis 1999;29:253-8.

2. Wey SB, Mori M, Pfaller MA, Woolson RF, Wenzel RP. Hospitalacquired candidemia. The attributable mortality and excess length of stay. Arch Intern Med 1988;148:2642-5.

3. Pfaller MA. Nosocomial candidiasis: Emerging species reservoir and modes of transmission. Clin Infect Dis 1996;22:S89-94.

4. Pittet D, Wenzel RP. Nosocomial blood stream infection. Secular trends in rates, mortality, and contribution to total hospital deaths. Arch Intern Med 1995;155:1177-84.

5. Wingard JR. Importance of Candida species other than C. albicans as pathogens in oncology patients. Clin Infect Dis 1995;20:115-25.

6. Bross J, Talbot GH, Maislin G, Hurwitz S, Strom BL. Risk factors of nosocomial candidemia: A case- control study in adult without leukemia. Am J Med 1989;87:614-20.

7. Wey SB, Mori M, Pfaller MA, Woolson RF, Wenzel RP. Risk factors for hospital-acquired candidemia: A matched case control study. Arch Intern Med 1989;149:2349-53.

8. Lecciones JA, Lee JW, Navarro EE, Witebsky FG, Marshall D, Steinberg SM, et al. Vascular catheter associated fungemia in patients with cancer: Analysis of 150 episodes. Clin Infect Dis 1992;14:875-83.

9. Karabinis A, Hill C, Leclercq B, Tancrede C, Baume D, Andremont A. Risk factors for candidemia in cancer patients: A case control study. J Clin Microbiol 1988;26:429-32.

10. Kao AS, Brandt ME, Pruitt WR, Conn LA, Perkins BA, Stephens DS, et al. The Epidemiology of candidemia in Two United States Cities: Results of a population-based active surveillance. Clin Infect Dis 1999;29:1164-70.

11. Rees JR, Pinner RW, Hajjeh RA, Brandt ME, Reingold AL. The epidemiological features of invasive mycotic infections in the San Francisco Bay area, 1992-1993: Results of population-based laboratory active surveillance. Clin Infect Dis 1998;27:1138-47.

12. Petrosillo N, Viale P, Nicastri E, Arici C, Bombana E, Caella A, et al. Nosocomial blood stream infections among human immunodeficiency virus infected patients: Incidence and risk factors. Clin Infect Dis 2002;34:677-85.

13. Launay O, Lortholary O, Bouges-Michel C, Jarrousse B, Bentata M, Guillevin L. Candidemia: A nosocomial complication in adults with late-stage AIDS. Clin Infect Dis 1998;26:1134-41.

14. Dato VM, Dajani AS. Candidemia in children with central venous catheters: Role of catheter removal and amphotericin B therapy. Pediatr Infect Dis 1990;9:303-14.

15. Edwards JE Jr, Bodey GP, Bowden RA, Buchner T, de Pauw BE, Filler SG, et al. International conference for the development of consensus on the management and prevention of severe candidal 
Infections. Clin Infect Dis 1997;25:43-59.

16. Nucci M, Colombo Al, Silveira F, Richmann R, Saloma R, Branchini $\mathrm{ML}$, et al. Risk factors for death in patients with candidemia. Infect Control Hosp Epidemiol 1998;19:846-50.

17. Silvestri L, delaCal MA, Von Saene HK, et al. Management of coagulase-negative staphylococcal, enterococcal and fungal problem in the intensive care unit. In: Von Saene HK, Silvestri L, dela Cal $M A$, editors. Infection control in the intensive care unit. SpringerVerlag Italia: Milan; 1998. p. 341-50.

18. van Saene R, Fairclough S, Petros A. Broad and narrow spectrum antibiotics: A different approach. Clin Microbiol Infect 1998;4:56-7.

19. Hope W, Morton A, Eisen DP. Increase in prevalence of nosocomial non-Candida albicans Candidaemia and the association of Candida krusei with fluconazole use. J Hosp Infect 2002;50:56-65.

20. Borg-von Zeppelin M, Eiffert H, Kann M, Ruchel R. Changes in the spectrum of fungal isolates: Results from clinical specimens gathered in 1987/88 compared with those in 1991/92 in the university of Gottingen, Germany. Mycoses 1993;36:247-53.

21. Abi-Said D, Anaissie E, Uzun O, Raad I, Pinzcowski H, Vartivarian $\mathrm{S}$. The epidemiology of hematogenous candidiasis caused by different Candida species. Clin Infect Dis 1997;24:1122-8.

22. Rees JR, Pinner RW, Hajjeh RA, Brandt ME, Reingold AL. The epidemiological features of invasive mycotic infections in the San Francisco Bay area, 1992-1993: Results of population-based laboratory active surveillance. Clin Infect Dis 1998;27:1138-47.

23. Diekema DJ, Messer SA, Brueggemans AB, Coffman SL, Doern GV, Herwaldt LA, Epidemiology of candidemia: 3-year results from the emerging infections and the epidemiology of lowa organisms study. J Clin Microbiol 2002;40:1298-302.

24. Sandven P, Bevanger L, Digranes A, Gaustad P, Haukland HH, Steinbakk M. Constant low rate of fungemia in Norway, 1991 to 1996. J Clin Microbiol 1998;36:3455-9.

25. Leleu G, Aegerter P, Guidet B; College des Utilisateurs de Base de Donnees en Reanimation. Systemic candidiasis in intensive care units: A multicentre, matched cohort study. J Crit Care 2002;17:168-75.

26. Gudlauqsson O, Gillespie S, Lee K, Vande Berg J, Hu J, Messer $\mathrm{S}$, et al. Attributable mortality of nosocomial candidemia, revisited. Clin Infect Dis 2003;37:1172-7.

27. Rex JH, Walsh TJ, Sobel JD, Filler SG, Pappas PG, Dismukes WE, et al. Practice guidelines for the treatment of candidiasis. Infectious Diseases Society of America. Clin Infect Dis 2000;30:666-78.

28. Maschmeyes G, Ruhnke M. Update on antifungal treatment of invasive Candida and Aspergillus infection. Mycoses 2004;47:26376.

29. Denning DW. Echinocandins: A new class of antifungals. J Antimicrob Chemother 2002;49:889-91.

30. Slavin MA, Osborne B, Adams R, Levenstein MJ, Schoch HG, Feldman AR, et al. Efficacy and safety of fluconazole prophylaxis for fungal infections after marrow transplantation-a prospective, randomized, double-blind study. J Infect Dis 1995;171:1545-52.

Source of Support: Nil, Conflict of Interest: None declared

\section{Author Help: Reference checking facility}

The manuscript system (w ww.journalonw eb.com) allows the authors to check and verify the accuracy and style of references. The tool checks the references with PubM ed as per a predefined style. Authors are encouraged to use this facility before submitting articles to the journal.

- The style as well as bibliographic elements should be $100 \%$ accurate to get the references verified from the system. A single spelling error or addition of issue number / month of publication will lead to error to verifying the reference.

- $\quad$ Example of a correct style

Sheahan P, O'leary G, Lee G, Fitzgibbon J. Cystic cervical metastases: Incidence and diagnosis using fine needle aspiration biopsy. Otolaryngol Head Neck Surg 2002;127:294-8.

- $\quad$ Only the references from journals indexed in PubM ed w ould be checked.

- $\quad$ Enter each reference in new line, without a serial number.

- $\quad$ Add up to a maximum 15 reference at time.

- If the reference is correct for its bibliographic elements and punctuations, it will be show $n$ as CORRECT and a link to the correct article in PubM ed will be given.

- If any of the bibliographic elements are missing, incorrect or extra (such as issue number), it will be show $n$ as INCORRECT and link to possible articles in PubM ed will be given. 